\title{
Entre techos de cristal y nichos académicos: estado actual de las mujeres en la arqueología peruana
}

\section{Between glass ceilings and research niches: The current situation of women in Peruvian archaeology}

\section{Lady Santana Quispe ${ }^{1}$}

Universidad de Rennes 1. Rennes, Francia

ladysantana04@gmail.com

ORCID 0000-0002-8980-2215

Citar como: Santana, L. (2019). Entre techos de cristal y nichos académicos: estado actual de las mujeres en la arqueología peruana. Desde el Sur, 11(2), pp. 261-281.

\section{RESUMEN}

Este artículo tiene como objetivo evaluar y caracterizar el estado actual de las mujeres dentro de la praxis arqueológica peruana y, de esta manera, revelar la existencia del denominado techo de cristal y la creación de nichos académicos. Como parte de la metodología realizamos un análisis cuantitativo y comparativo del número de ingresantes, matriculados y egresados de Arqueología durante 2014. Utilizamos también los registros de docentes en cada una de las planas docentes y, finalmente, hicimos una revisión de las seis ediciones del Congreso Nacional de Arqueología. Los resultados nos indican que el estado actual de las mujeres en la arqueología continúa siendo de desigualdad y subrepresentación. Además, se evidencia la existencia del denominado techo de cristal y el desarrollo de nichos académicos.

\section{PALABRAS CLAVE}

Mujeres, arqueología, techo de cristal, nichos académi$\cos$

1 Lady Santana Quispe es arqueóloga egresada de la Universidad Nacional Mayor de San Marcos y beneficiaria en 2016 de la beca integral Fondecyt Perú para estudios de posgrado. Es magíster, con enfoque arqueométrico, por la Universidad de Rennes 1 (2018). Ha realizado investigaciones geoarqueológicas en Samaca, en la región Ica, lugar desde donde aborda problemáticas como el cambio medioambiental y la transformación del paisaje. Asimismo, realiza trabajos enfocados en temas de género, en especial sobre la praxis arqueológica. 


\section{ABSTRACT}

The aim of this article is to evaluate and characterize the current status of women within the Peruvian archaeological community and thus confirm the existence of the so-called glass ceiling and the creation of academic niches. Our methodology incorporates a quantitative and comparative analysis of the numbers of candidates, students and graduates within the field of archaeology during 2014. In addition, we examined the teaching staff records for individual institutions, and finally we reviewed the six annual events of the National Congress of Archaeology. Our results indicate that the current status of women in archaeology continues to be defined by inequality and underrepresentation, while confirming the existence of a so-called glass ceiling and the formation of academic niches.

\section{KEYWORDS:}

Women, Peruvian archaeology, glass ceiling, academic niches

\section{Introducción}

La condición de las mujeres en distintas actividades de la vida humana ha mejorado con el transcurso de los años en aspectos públicos y educativos (Peppino, 2006, p. 3), especialmente en su participación en las ciencias y tecnologías, campos donde estuvieron, no hasta hace mucho tiempo, alejadas, relegadas $y$, en ocasiones, invisibilidades por ser consideradas cosas de «hombres» (Puertas, 2015). Afortunadamente, esta tendencia mundial ha cambiado y continúa en proceso de transformación, a través de la inserción e inclusión de las mujeres en campos científicos, académicos y profesional (para mayor detalle, ver Puertas, 2015). En el caso de Europa, en especial las universidades de España, Reino Unido, Alemania y Países Bajos, el número de estudiantes mujeres varía entre $54 \%$ al $59 \%$ del total de estudiantes, desde inicios del siglo XXI hasta 2010. En el ámbito latinoamericano, en países como Brasil y México, los de mayor extensión geográfica, la representación porcentual de mujeres y hombres varía entre el $48 \%$ al $50 \%$, respectivamente, y algunas veces presentan porcentajes equitativos (Guil, 2008).

Sin embargo, la situación cambia cuando se produce el egreso de estudiantes. El ámbito laboral, académico y profesional son distintos, y la balanza empieza a inclinarse a favor de los hombres (Guil, 2008). Esta situación ha sido analizada desde la década de 1980, cuando se aborda la problemática a través del reconocimiento de la existencia del llamado 
techo de cristal (Guil, 2008, p. 213), que representa las barreras, a menudo «invisibles», que persisten en los ámbitos mencionados y dificultan el acceso de las mujeres a espacios de prestigio, poder y a un mejor salario. Este término tiene su origen en un artículo de Hymowirtz y Scheldart de 1986, publicado en Wall Street Journal (Bastida, 2018).

Estas barreras, nos menciona Bastida (2018), pueden ser externas, es decir, aquellas que no son innatas a la mujer, sino que son propiciadas por el entorno (por ejemplo, los estereotipos de género o cultura organizacional). Asimismo, pueden ser internas y estar relacionadas a decisiones personales, características de personalidad y estereotipos de género. Finalmente, estas barreras pueden ser mixtas, y estar ligadas a temas de maternidad, distribución de roles domésticos, falta de apoyo organizacional y social.

Esta situación, a su vez, genera la existencia de los llamados nichos académicos, los cuales se originan por la tendencia de las mujeres a participar de espacios y temas de investigación específicos, como resultado de estrategias de legitimación, identidad y recurso (Gastiazoro, 2013; Heller, 2012), que les permiten un mejor desarrollo y búsqueda de comodidad. El nicho académico se puede ejercer y percibir tanto de manera consciente y directa, como inconsciente e indirecta, por las pautas que marcan los espacios laborales y académicos a través del tiempo (Guil, 2008).

En el caso peruano, las ciencias en general, y las sociales y humanas en particular, vienen experimentando esta tendencia de incorporación de mujeres en los ámbitos universitarios, académicos y profesionales (aunque estos dos últimos en menor medida) (Sunedu, 2019; Alcázar y Balarin, 2018). Esta llamada feminización de las aulas (Alcázar y Balarin, 2018) se ve reforzada por la presencia de las mujeres en las principales universidades públicas y privadas del Perú de 2014 a 2016, periodo en el cual se percibe un incremento sustancial en las cifras de mujeres universitarias (varían entre el $45 \%$ al $52 \%$ del total de estudiantes universitarios) (Sunedu, 2019). No obstante, esto no representa la distribución numérica de mujeres por cada carrera universitaria, la cual podría variar, por ejemplo, en áreas relacionadas con las ingenierías, de acuerdo con el patrón histórico de recurrencia.

Sin embargo, la situación va cambiando cuando tanto mujeres como hombres finalizan sus carreras profesionales y se enfrentan a espacios laborales, académicos y de toma de decisiones. Un ejemplo a citar es el trabajo publicado en 2017 por el Consejo Nacional de Ciencia Tecnología e Innovación Tecnológica (Concytec), que presentó los resultados del I Censo Nacional de Investigación y Desarrollo de Centros de Investigación de 2016. En el documento se muestra que en 2015, del total de 
investigadores, el 68,1\% (representado por 2298 investigadores) eran hombres y el 31,9\% (1074 investigadores) eran mujeres (Concytec, 2017). Esto muestra una amplia e importante brecha entre investigadores e investigadoras del Perú.

La arqueología, como una ciencia social y humana, no está exenta de estas recurrencias, situaciones y problemáticas, por lo que recurrimos a una de las dos perspectivas que se desarrollan en arqueología de género para estudiar la situación de la mujer. El primer criterio se relaciona con el estudio de la mujer como tópico principal en sí mismo, es decir, la mujer en el pasado. El segundo está dedicado a evaluar la situación y la relación de género en la práctica arqueológica. Este último es de interés de este artículo, cuyo objetivo es evaluar el estado de la cuestión de las mujeres en arqueología, desde una perspectiva de relación de género, que nos permita evidenciar la posible existencia del llamado techo de cristal y nichos académicos en el ámbito arqueológico peruano.

Para ello, nos valemos de un análisis cuantitativo de la presencia de mujeres y hombres en las universidades que imparten la carrera de Arqueología, en las planas docentes de estas universidades y de la participación de ellos en el Congreso Nacional de Arqueología (CNA), como un espacio académico dedicado a reunir una gran cantidad de investigaciones anuales.

A través de las páginas de este documento veremos algunos aspectos vinculados al género, la ciencia y la arqueología de género, así como la metodología empleada, los resultados graficados y representados en tablas, la interpretación que correlaciona las categorías techo de cristal y nichos académicos, y, finalmente, nuestras conclusiones.

\section{Ciencia, género y arqueología de género}

De acuerdo con Cristina Bellelli, Mónica Berón y Vivian Scheinsohn, tanto ciencia como género son categorías construidas social y culturalmente (1993, p. 47). El género, nos dice Ruth Falcó:

identifica lo que es socialmente esencial en el hecho de ser varón y mujer. Lo biológico, el sexo, es perceptible externamente y no varía en las diferentes sociedades, mientras que lo social, el género, varía en cada sociedad (2003, p. 64).

La autora explica que el término género se refiere a las relaciones entre hombres y mujeres, y cómo estas son socialmente construidas (Falcó, 2003 , p. 64). Por otro lado, las relaciones entre género y ciencia pueden ser abordadas desde dos perspectivas (Bellelli et al., 1993): 1) desde el criterio epistemológico, que evalúa la construcción y la producción del 
conocimiento científico como tal, y 2) desde la forma en que estas categorías se reflejan en la práctica científica.

En el caso de la arqueología, en especial la arqueología de género a partir de las tres últimas décadas del siglo pasado, empezó un movimiento teórico-metodológico crítico que evaluó las presunciones androcéntricas arraigadas en las interpretaciones sesgadas de las sociedades del pasado (Conkey, 2003; Conkey y Spector, 1984; Gero, 1991), con lo cual se tiende a legitimar la situación actual.

Dentro de este contexto, las mujeres en arqueología han adoptado por seguir dos vías. La primera se dedica al proceso de visibilización de las mujeres en la historia de la humanidad, y la segundo, y punto de partida de este artículo, es el camino que conduce a analizar las relaciones de género en la práctica arqueológica. Nuestro aporte se encuadra en esta perspectiva.

Aunque en arqueología la mayoría de trabajos se vincula a la primera visión, y hasta se crean corrientes generales como en España (Sánchez, 2000; Díaz-Andreu, 1998) o Estados Unidos (Conkey, 2003; Conkey y Spector, 1984; Gero 1991), los trabajos con enfoque relacionados con la segundo perspectiva son escasos (Bellelli et al., 1993; Gero 1988) o se hace mención de manera superficial (Díaz-Andreu 2013; Conkey y Spector, 1984). La arqueología en el Perú ha abordado temas adscritos a la primera perspectiva bajo el paraguas de la arqueología de género (Chacaltana, 2109, 2016).

En cuanto a la segunda perspectiva, durante los últimos seis o siete años esta visión ha ido encontrando un espacio, aunque pequeño, en la arqueología peruana. De alguna manera se llamó la atención de empezar la historia de la arqueología peruana de manera integral y completa (DíazAndreu, 2013). Así, algunos aportes han aparecido en este ámbito, como los de Pedro Novoa (2013), quien, si bien no hace un análisis crítico de las relaciones de género en la práctica arqueológica peruana, sí nos presenta información relevante en cuanto al contexto en el que Rebeca Carrión Cachot participó y empezó a colocar los cimientos de la arqueología en el Perú.

Por otro lado, Claudia Núñez (2018), en un manifiesto, en parte autobiografiado, presentado en el simposio «Mujeres en la arqueología: pasado, presente y futuro. Contribuciones a la praxis arqueológica en el Perú», llevado a cabo en el Museo de Arqueología, Antropología e Historia del Perú en agosto de 2018, nos exclama que hay mujeres en arqueología, pero que son a menudo invisibilidades. Sin embargo, su presencia ha obligado a que la disciplina se transforme. 
Asimismo, Tavera y Santana (2018), en una ponencia presentada en la I Jornada de Estudios de Género y Mujeres en la Historia del Perú, llevada a cabo en octubre de 2018, a través de un análisis bibliométrico, analizan las relaciones de género que las mujeres enfrentan al interior de la academia peruana. Para ello, se valieron de la información cuantitativa que les otorgó la revisión de dos de las revistas más representativas den al campo académico peruano: Arqueología y Sociedad de la Universidad Nacional Mayor de San Marcos y el Boletín de Arqueología de la Pontificia Universidad Católica del Perú. Las autoras hacen evidente la subrepresentación de mujeres en trabajos de publicación y permiten visibilizar la asimetría existente en esta ciencia.

Este proceso de surgimiento de estudios enfocados en la práctica arqueológica es gradual (Arroyo, 2019 en este volumen; Cabrera, 2019 en este volumen; Pacheco, 2019 en este volumen; Tavera, 2019 en este volumen). Sin embargo, es necesario responder a diversas preguntas; entre ellas, cuántas mujeres ingresan a la carrera de Arqueología, cuántas se mantienen estudiando en las universidades, cuál es la característica de esta nueva presencia, cuántas se dedican a la investigación científica, qué tipo de espacios académicos ocupan o generan, cuántas se encargan de formar nuevos arqueólogos, entre otras preguntas que de manera directa e indirecta tratamos de expresar en este artículo.

\section{Metodología}

En este trabajo realizamos un análisis cuantitativo-comparativo de la presencia de mujeres en la arqueología, desde su ingreso a la carrera, su permanencia, su egreso y el desempeño en el campo académico-profesional. Para este fin, analizamos los datos proporcionados por la Superintendencia Nacional de Educación Superior Universitaria (Sunedu).

En este punto es importante indicar que la Sunedu ${ }^{2}$ cuenta con información estadística - a partir de 2014- de las universidades licenciadas y facultades que cumplen con el proceso de acreditación. Por tanto, en este análisis no se cuenta con información de las 10 universidades que ofrecen la carrera de Arqueología en el Perú (tabla 1).

TABLA 1. Universidades con la carrera profesional de Arqueología

\begin{tabular}{ll} 
Universidades & Tipo de información (2014) \\
\hline Universidad Nacional Federico Villareal & Ingresantes - No cuenta - No cuenta \\
\hline Universidad Nacional Santiago Antúnez de Mayolo & Ingresantes - Matriculados - Egresados \\
\hline
\end{tabular}

2 Creada en el marco de la promulgación de la Ley Universitaria 30220. 


\begin{tabular}{lll}
\hline Universidad Nacional San Antonio Abad de Cusco & Ingresantes - Matriculados - Egresados \\
\hline Universidad Nacional San Cristóbal de Humanga & $\frac{\text { Ingresantes - No cuenta - No cuenta }}{\text { Universidad Nacional Toribio Rodríguez de Mendoza }}$ & $\frac{\text { Ingresantes - Matriculados - No cuenta }}{\text { Ingresantes - Matriculados }}$ \\
\hline Universidad Nacional de Trujillo & $\frac{\text { Ingresantes - Matriculados - Egresados }}{\text { Universidad Nacional Mayor de San Marcos }}$ & Ingresantes - Matriculados - Egresados \\
\hline Pontificia Universidad Católica del Perú & La tabla muestra las universidades que son parte del análisis cuantitativo y el tipo de información que han proporcionado \\
en 2014
\end{tabular}

La revisión de las planas de docentes nombrados registrados en seis universidades que ofrecen la carrera de Arqueología (en el Perú, nueve universidades públicas y una una privada ofrecen este programa): Estas universidades son la Universidad Nacional de Trujillo (UNT), la Universidad Nacional Santiago Antúnez de Mayolo ${ }^{3}$ (Unsam), la Universidad Nacional Mayor de San Marcos (UNMSM), la Universidad Nacional San Luis Gonzaga de Ica (Unica), la Universidad Nacional San Antonio de Abad del Cusco (Unsaac) y la Pontificia Universidad Católica del Perú (PUCP). Esta información está dividida en las siguientes categorías: profesor auxiliar, profesor asociado y profesor principal, con lo cual podremos identificar un posible techo de cristal.

También realizamos otro análisis cuantitativo-comparativo de la presencia de mujeres en espacios académicos y de divulgación. Para ello, seleccionamos las actas y sumillas (IV, V y VI edición) del CNA. Elegimos el CNA, desde su primera edición en 2014, debido a que este espacio tiene como objetivo principal reunir los trabajos arqueológicos realizados en el territorio peruano en un periodo anual, por arqueólogos nacionales o extranjeros. Este análisis nos permitirá ver cuántas mujeres participan a través de ponencias y su subsecuente publicación, además de conocer en qué campos de la disciplina tienen mayor, menor o igualdad de presencia, lo que nos llevará por el camino de la identificación de un posible techo de cristal y de nichos académicos.

Debemos puntualizar que usaremos la categoría de autores, autoras, mujeres y hombres. En el caso de las dos primeras, es preciso señalar que el número de autores es diferente al número de firmas. El primero está directamente relacionado con la persona que publica (Agut, 2016), por lo que es posible encontrar dos o más trabajos con el mismo autor en una misma edición del CNA. Esta situación es beneficiosa para los objetivos de este artículo, en tanto nos permitirá ver la distribución multifacética de un arqueólogo

3 En este caso solo existe un nombramiento. Sin embargo, dentro de los contratados no se registra ninguna mujer. 
y establecer nichos de investigación. Con esta finalidad se han identificado temas recurrentes en seis ediciones (2014-2019) del CNA. Subsecuentemente, se hizo un listado y se abreviaron para ser representados en gráficas estadísticas mostradas en la sección «Resultados» (tabla 2 y figura 1).

TABLA 2. Temas identificados en las seis ediciones del CNA (2014-2019)

\begin{tabular}{|c|c|c|}
\hline Tema & Tema & Contenido \\
\hline Arqueología de contrato y rescate & ArqCon-Res & $\begin{array}{l}\text { Presentación de proyectos y trabajos de evaluación, moni- } \\
\text { toreo y rescate }\end{array}$ \\
\hline Arqueología en general & ArqGe & $\begin{array}{l}\text { Todo lo concerniente a la explicación de procesos, presenta- } \\
\text { ción de datos de excavación, prospección, caracterización de } \\
\text { un sitio, periodo, etc. }\end{array}$ \\
\hline Arqueología de género & ArqGene & Aplicación de la teoría de género \\
\hline Arqueología histórica & ArqHis & Arqueología desde el periodo colonial y republicano \\
\hline Arqueometría & ArqMe & $\begin{array}{l}\text { Aplicación de métodos y técnicas de ciencias básicas, natu- } \\
\text { rales y estadísticas-comunicación en la arqueología con la } \\
\text { arqueología }\end{array}$ \\
\hline Arqueología de la muerte & ArqMue & $\begin{array}{l}\text { Análisis de contextos funerarios, patrón funerario, cemente- } \\
\text { rios arqueológicos, etc. }\end{array}$ \\
\hline $\begin{array}{l}\text { Arquitectura y arqueología del pai- } \\
\text { saje }\end{array}$ & ArqPai & $\begin{array}{l}\text { Análisis espacial, arquitectónico, patrón de asentamiento, } \\
\text { etc. }\end{array}$ \\
\hline Análisis de material orgánico & AM0 & $\begin{array}{l}\text { Análisis de materiales como restos macrobotánicos, malaco- } \\
\text { lógicos, zooarqueológicos }\end{array}$ \\
\hline Análisis ceramográfico & AnCe & Análisis tradicional de cerámica arqueológica \\
\hline Análisis textil & AnTex & Análisis tradicional de tejidos y textiles arqueológicos \\
\hline Aportes teórico-metodológicos & ATM & $\begin{array}{l}\text { Propuestas teóricas metodológica para el estudio y la ges- } \\
\text { tión del patrimonio }\end{array}$ \\
\hline Bioarqueología & Bioarq & Osteología \\
\hline Gestión del patrimonio & $\mathrm{GePa}$ & $\begin{array}{l}\text { Puesta en valor, museos, educación, plan de manejo cultu- } \\
\text { ral, relaciones comunitarias, etc. }\end{array}$ \\
\hline Multidisciplinar & Multidi & Más de dos objetos en estudio \\
\hline
\end{tabular}

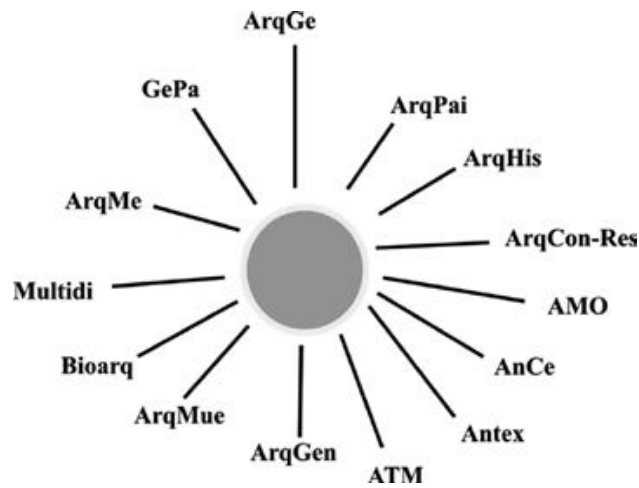

FIGURA 1. Temas identificados de acuerdo con temáticas planteadas durante las seis ediciones del CNA. 


\section{Resultados}

¿Cuántas mujeres ingresan a la carrera de Arqueología? ¿Cuántas están matriculadas? ¿Cuántas egresan? ¿Cuántas se desempeñan como docentes?

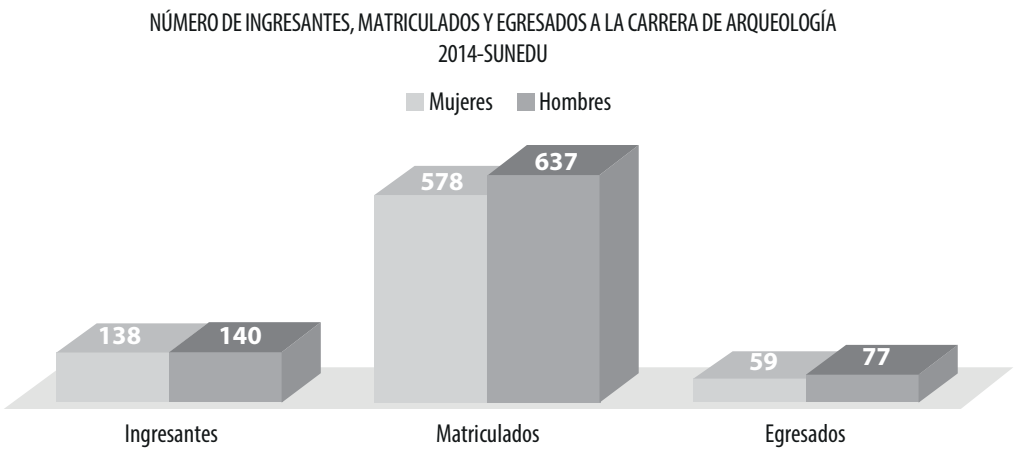

FIGURA 2. Gráfica de barras que muestra las cantidades de ingresantes, alumnas matriculados y egresados a la carrera de Arqueología.

La figura 2 muestra la cantidad de estudiantes que ingresaron a la carrera de Arqueología en 2014 en ocho de las universidades que imparten esta profesión. Los números representan cierta paridad, lo cual indica el acceso equitativo a las aulas universitarias, y también «igualdad» de oportunidades. Asimismo, la gráfica muestra la cantidad de estudiantes matriculados para 2014 en seis de las universidades que ofrecen el programa de Arqueología. Aquí, la distribución por sexo es diferente, ya que las mujeres representan el $48 \%$ del total de matriculados. Estos números muestran una brecha corta en cuanto a hombres y mujeres, lo cual indica que en la actualidad la arqueología, en el pregrado, no es una carrera masculinizada.

La figura representa también las cantidades de egresados durante el mismo año en cuatro de las universidades del país que imparten la carrera. Aquí, la situación es distinta, pero no drásticamente diferente, en tanto que las mujeres representan el $43 \%$ del total de egresados. Sin embargo, la tendencia se encamina hacia la ampliación de la brecha entre hombres y mujeres en el proceso de formación universitaria. En general, se observa que, en el inicio de este proceso, el escenario es aparentemente igualitario, pero cambia cuando se llega a la etapa de egreso y comienza la ampliación de la brecha de desigualdad de género. Estas cifras ayudan a reafirmar la evidente feminización de las aulas.

Una vez obtenido el grado académico y la licenciatura, los lugares donde se desarrolla la práctica arqueológica se concentran en la docencia, 
los proyectos de investigación, el Ministerio de Cultura, entre otros ámbitos. En este caso analizaremos la plana docente de las universidades.

La figura 3, por su parte, muestra la cantidad de docentes de planta en seis universidades, con datos actualizados a 2019 (UNT, Unsaac, PUCP, Unsam, UNMSM y Unica). Se observa una amplia diferencia en cuanto a la presencia de mujeres impartiendo clases en universidades, pues representan el $40 \%$ del total de docentes ( 19 registradas), frente a un $60 \%$ de hombres ( 28 registrados). Aunque en total el número parece reducido, es mayor la cantidad de profesores en las universidades, ya que se encuentran bajo la modalidad de contrato, lo que dificulta su identificación para fines de este estudio.

La figura 4 muestra la distribución numérica de docentes por categorías y sexo en seis de las universidades seleccionadas (UNT, Unsaac, PUCP, Unsam, UNMSM y Unica). Las columnas reflejan, mediante categorías de docentes, la preponderancia de hombres en todas las planas docentes analizadas y representadas en la figura 3. En detalle, en el gráfico de barras (figura 4) las mujeres se ven mejor representadas numéricamente (nueve registradas) en la categoría «profesora auxiliar», superando a sus pares hombres (cinco registrados), por lo que representan el $60 \%$ del total ( 14 registrados) de esta categoría.

TOTAL DE PROFESORAS/ES EN LAS UNIVERSIDADES SELECCIONADAS

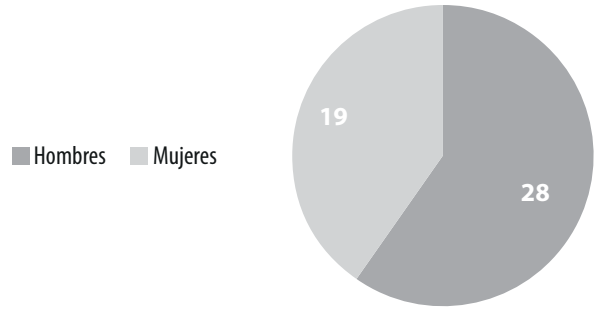

FIGURA 3. Cantidad de docentes mujeres y hombres en seis universidades del Perú.

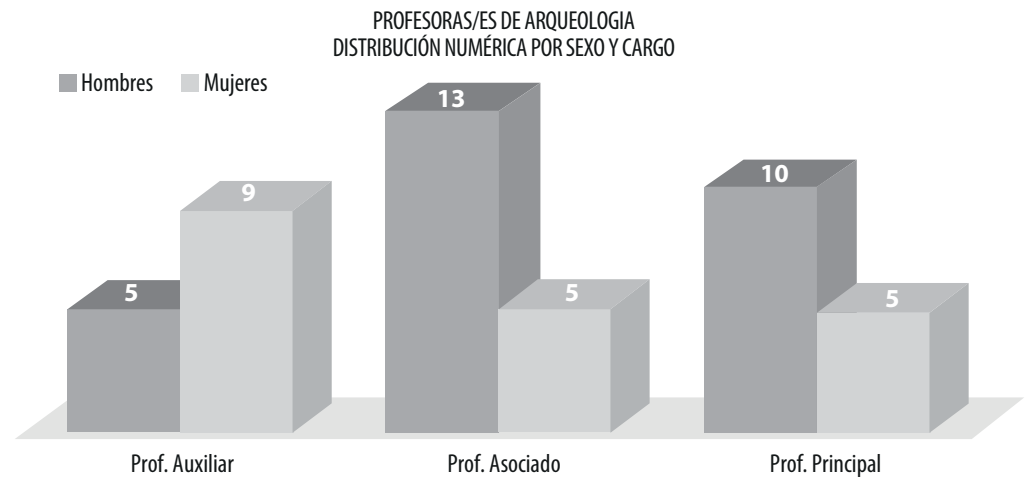

FIGURA 4. Distribución numérica de profesores de Arqueología en seis universidades del país. 
La situación cambia a mitad de estos niveles. En la categoría «profesores asociados», los docentes arqueólogos se ven mejor representados numéricamente (13 registrados), frente a una subrepresentación de sus pares mujeres (5), quienes constituyen solo el $28 \%$ del total de registros (18), ante un $72 \%$ de hombres registrados. El escenario es el similar en la categoría «profesores principales»: en este nivel, los hombres representan el $66 \%$ del total de registros en esta categoría (15), y las mujeres un $23 \%$.

Los datos antes presentados dan cuenta de un análisis general de las planas docentes. Sin embargo, si realizamos una revisión más exhaustiva de cada una, se observan cifras numéricas dramáticamente desiguales. La figura 5 refleja estos detalles.

La figura 5 muestra ausencia de docentes mujeres en algunas universidades como la Unsam, donde solo se registró a un profesor principal. En contraste, la UNMSM y la Unsaac presentan una mejor distribución de profesores y profesoras en su plana docente, al tener 16 y 10 docentes registrados, respectivamente. Por otro lado, la presencia de mujeres en los niveles superiores es escasa en el caso de la UNMSM y la Unica. La situación es excepcional: se presentan dos casos de representación de 2 hombres y 2 mujeres, y 1 mujer y 1 hombre, respectivamente. No obstante, la Unica solo registra una docente mujer. El escenario cambia drásticamente en la PUCP, en donde el total de profesores principales está conformado por hombres (6) y nula presencia de mujeres en esta categoría. Además, en la PUCP solo se han registrados profesores principales y profesoras auxiliares, grupo representado en su totalidad por mujeres (2).

DISTRIBUCIÓN DE PROFESORAS/ES POR CATEGORÍA EN 6 UNIVERSIDADES SELECCIONADAS

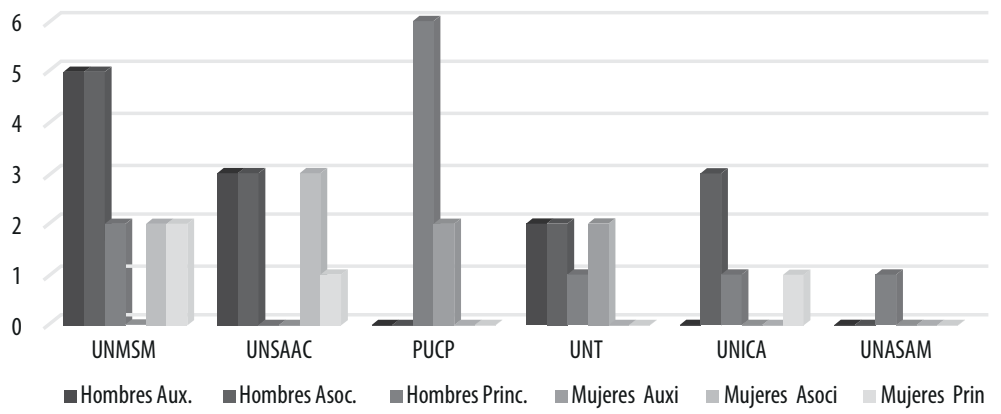

FIGURA 5. Gráfico de distribución de docentes hombres-mujeres/categoría por cada universidad.

A pesar de la alta presencia de docentes mujeres en la categoría «profesor auxiliar», en la UNMSM y la Unsaac es nula la presencia de mujeres en este nivel. Predomina la presencia absoluta de hombres, en contraste con universidades como la UNT o la PUCP, en donde las mujeres representan la mitad (2) o el total (2) de docentes registrados en este nivel. En 
cuanto a profesores de la categoría «asociados», la UNMSM y la Unsaac son las únicas universidades que registran presencia de mujeres. En la Unsaac la relación de paridad entre hombres y mujeres se hace evidente: 3 y 3 registros, respectivamente.

¿Cuál es el campo de investigación de las mujeres arqueólogas? ¿Qué investigan las mujeres arqueólogas?

En relación con esta categoría planteada, cuyo análisis recae en el CNA, obtuvimos que desde el inicio del CNA (tabla 3), en 2014 (I CNA), hasta la edición de 2019 (VI CNA), existen 460 ponencias distribuidas en 14 temas. La cantidad de autorías en los seis años es de 895, con presencia de 367 mujeres y 528 hombres. La figura 6 representa la cantidad de hombres y mujeres, y se observa una marcada diferencia - 161 autorías de diferencia- en cuanto a participación de arqueólogas en este campo académico, con un total de 367 autorías, frente a 528 autorías de arqueólogos ( $41 \%$ y $59 \%$, respectivamente) (figura 7 ).

TABLA 3. Datos generales de las ediciones del CNA

Número de ponencias, temas, autorías de mujeres y hombres registradas para las seis ediciones del CNA

\begin{tabular}{|c|c|c|c|c|c|}
\hline Año de edición & Ponencias & Temas & Autorías & Autoría mujeres & Autoría hombres \\
\hline $2014-2019$ & 460 & 14 & 1165 & 367 & 528 \\
\hline
\end{tabular}

La figura 8 muestra la tendencia de participación de mujeres y hombres en cada edición del CNA. De 2014 a 2019 la presencia de mujeres está representanda por una línea cuya característica en general es ascendente. El punto de quiebre se experimenta a partir de año 2018 (V CNA) y llega a su pico más alto en la última edición de 2019 (VI CNA). Para el caso de los hombres la tendencia muestra una mayor participación de arqueólogos a lo largo del tiempo con picos altos como 2014 y 2018.

TOTALAUTORAS/ES PRESENTES EN LAS 6 EDICIONES DELCNA

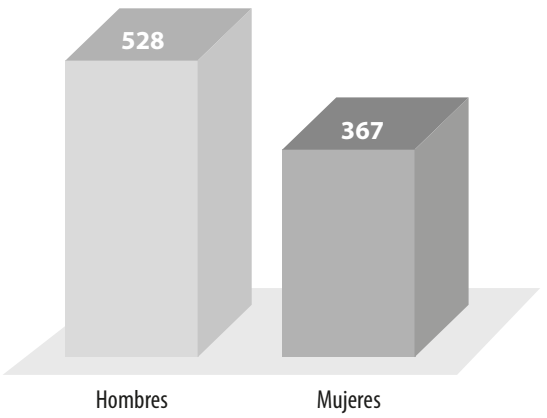

AUTORAS/ESEN LAS6EDICIONES DEL CNA

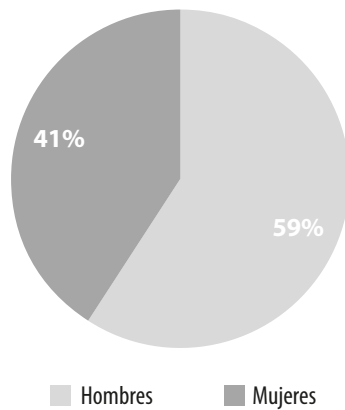

FIGURA 6 Y FIGURA 7. Número y cantidades porcentuales de autores presentes en las seis ediciones del CNA. 
Ambas líneas también nos muestran algunos detalles: por ejemplo, en 2017, la participación de mujeres y de hombres fue casi equitativa, a pesar de que en ambos casos se observa la reducción de la participación de autores.

Es importante señalar que en 2018 ambas líneas experimentan un proceso de ascendencia en el número de autorías. Las diferencias son drásticamente marcadas y superando en casi el 100\% la participación de arqueólogos frente a la de arqueólogas. Esta situación se revierte en 2019, ya que la participación de las mujeres supera por primera vez a la de hombres. Otras cifras reflejan que las diferencias no son marcadas en algunas de las ediciones del CNA (2015, 2016 y 2017). A pesar de ello, continúa el predominio de arqueólogos.

En cuanto a la distribución de mujeres y hombres en temas de ponencias, la figura 9 expone algunas recurrencias y tendencias que es necesario evidenciar. En las seis ediciones del CNA, la participación de arqueólogos en contenidos como arqueología general (297 autorías), arqueología de la muerte (27 autorías), arqueología del paisaje (57 autorías) y aportes teórico-metodológicos (27 autorías), se encuentran muy bien representados en la línea de distribución, frente a una marcada subrepresentación de las arqueólogas en estos campos de investigación.

La participación y distribución de las mujeres está bien constituido en ponencias relacionadas con la arqueometría (76 autorías), arqueología de contrato y rescate (9 autorías), análisis textil (6). Aunque las diferencias no son marcadas en comparación con los hombres, estas superan en número a sus pares arqueólogos. La línea de distribución también señala cierta participación «equitativa» de arqueólogos y arqueólogas en algunos temas como la arqueología de género (1-1), arqueología histórica (16-20 autorías), análisis de material orgánico (4-5 autorías), gestión del patrimonio (41-43 autorías) y el campo multidisciplinar (13-13 autorías), con números repartidos en $50 \%$ para cada uno en algunos casos (arqueología de género y multidisciplinar), o pequeñas diferencias porcentuales en otras (arqueología histórica, análisis de material orgánico y gestión del patrimonio).

\section{Discusión}

Los datos que aquí presentamos son útiles para discutir el estado de la cuestión de las mujeres arqueólogas en el Perú. Se han analizado y mostrado datos estadísticos de 2014 concernientes a 8 universidades de las 10 que ofrecen la carrera profesional de Arqueología. Estos datos, que exponen un escenario de aparente paridad con la presencia de 138 mujeres y 140 hombres, marcan la pauta para el inicio de un proceso de 
distintas etapas de transición; la primera, de formación, está caracterizada por una mayor inserción de las mujeres en la carrera de Arqueología, seguida por la permanencia, aunque con alguna diferencia numérica (578 mujeres matriculadas, frente a 637 hombres matriculados); al igual que en la etapa final, el egreso universitario (59 mujeres egresadas y 77 hombres egresados).

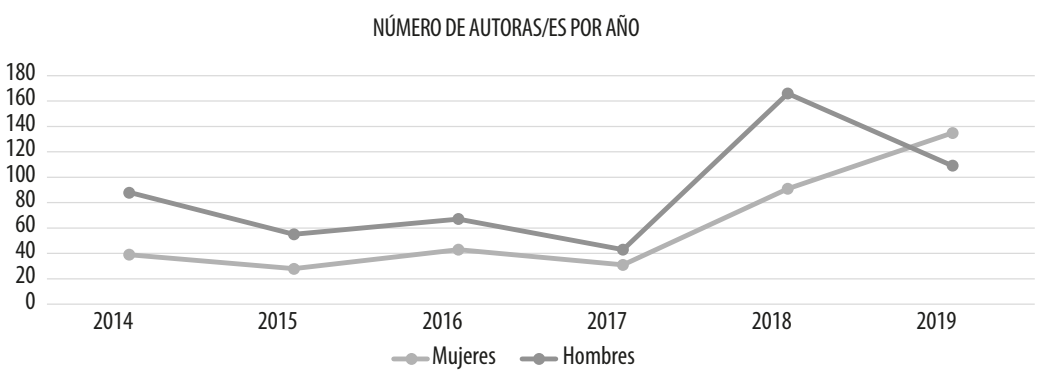

FIGURA 8. Gráfico de distribución de mujeres y hombres por año de participación en el CNA.

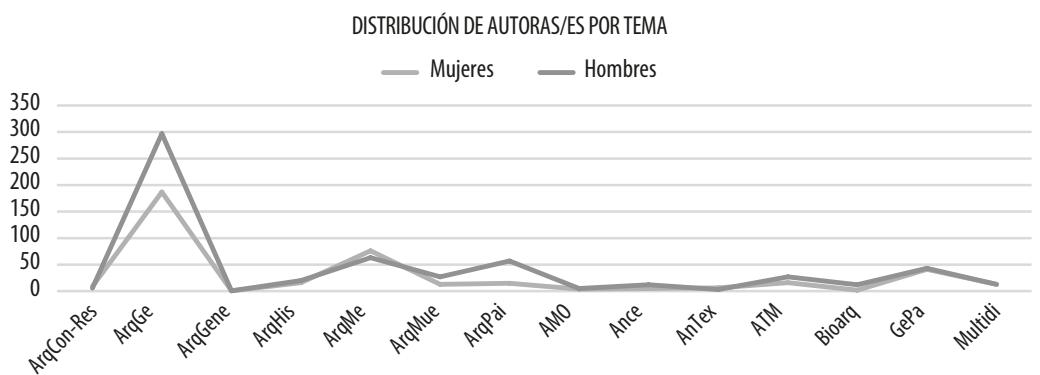

FIGURA 9. Gráfico de distribución de mujeres y hombres por temas de investigación.

Nos encontramos ante un escenario de aparente «igualdad» en estas primeras etapas de formación. No obstante, es necesario indicar que estos datos cuantitativos no expresan las relaciones de género cotidianas -el día a día- en las aulas y área laboral, ya que son difíciles de cuantificar sin testimonios directos. Es un tema que debe ser abordado con el mayor rigor posible.

En relación con el análisis de las planas docentes de las seis universidades revisadas (UNT, Unsam, UNMSM, PUCP, Unica y Unsaac), la información expuesta revela, a través de cifras (ver figura 3 ), la marcada diferencia entre la etapa de formación y la etapa de desarrollo profesional entre mujeres y hombres, la elevada presencia de estos últimos evidencia el limitado acceso de las mujeres a este campo laboral; la falta de políticas de paridad a nivel gubernamental como la Ley Universitaria; y, de manera específica, los reglamentos y estatutos de estas universidades (ver distribución 
de mujeres y hombres por universidades en figura 5). De igual manera, la supremacía específica de hombres en los niveles superiores (asociado y principal) y de mujeres en el nivel inicial (auxiliares) de la carrera docente (figura 4), permite ver que mientras más se avanza en instancias jerárquicas superiores, las mujeres dejan de ser el grupo mayoritario, y crean una marcada diferencia a favor de los hombres.

Estas condiciones originan al menos dos situaciones que vale la pena exponer: 1) que los cargos universitarios, la administración de poder y la toma de decisiones recaen mayoritariamente en los hombres y no permite una igual distribución de roles y participación entre hombres y mujeres; por ende, son ellos quienes normalmente deciden qué se imparte y cómo se organiza la carrera, qué se investiga y cómo se establece esta investigación; 2) la existencia de diferencias salariales, debido a la antagónica situación en la que se encuentran la mayoría de mujeres (en nivel inicialauxiliar) frente a la alta presencia de hombres en los niveles medios y superiores de la carrera. En este sentido, las mujeres perciben menor salario debido a que esta categoría, normalmente, es la de menor remuneración.

Esta primera parte del análisis da cuenta de la existencia de una pirámide cuya base tiende a ser equitativa durante la última década. Sin embargo, cuando avanzamos en los niveles más altos y jerárquicos, las mujeres disminuyen en su participación (UNMSM, Unica y Unsaac) e incluso desaparecen (PUCP, UNT y Unsam), lo que resulta en una marcada diferencia a favor de los hombres. Es, entonces, que empiezan a generarse y evidenciarse las consecuencias de aquellas barreras «invisibles» (techo de cristal), que aparentemente no se explican porque no existen. Por ejemplo, si efectuamos el ensayo de hablar con un grupo de arqueólogas y preguntarles si se sienten o se sintieron alguna vez discriminadas, relegadas o excluidas, probablemente las respuestas serán mayoritariamente negativas.

Supuestamente no existen restricciones, pero los datos cuantitativos muestran una realidad distinta, caracterizada por la presencia de barreras que afectan el desarrollo profesional de las mujeres y no les permiten cruzar con facilidad las vallas jerárquicas, y puedan progresar verticalmente. Estas barreras existen al margen de la formación de las mujeres, y es que si realizamos un ejercicio rápido de revisión de las planas docentes de las escuelas de Arqueología del Perú, un grupo mayoritario de mujeres poseen el grado de magíster o doctoras, principales requisitos (Ley Universitaria 30220) para acceder a la carrera docente o ascender en ella. En este punto es conveniente entonces cuestionarse cuál o cuáles son los motivos que generan este denominado techo de cristal. Quizá podamos encontrar algunas respuestas en la ausencia de políticas públicas de paridad laboral, 
la maternidad, el sesgo androcéntrico, las redes de contacto, el reparto de tareas domésticas, y la falta de ayuda sociales y organizacionales; en general, factores que se repiten en otras ciencias, y, como sugiere Bastida (2018), se agrupan en barreras externas, internas y mixtas, las cuales trascienden a nuestro contexto actual, se remontan a generaciones pasadas (Guil, 2008), y están muy arraigadas en nuestra sociedad patriarcal.

Por otro lado, el segundo análisis que ocupó este artículo reafirma esta tendencia de mayor presencia y participación de hombres en espacios de divulgación académica y profesional, problemática ya tratada y expuesta por Tavera y Santana (2018). A través de los seis años ininterrumpidos de edición del CNA, el gran número de intervencionez fue de hombres. Debemos destacar que quizá el fenómeno de feminización de las aulas pueda volcarse a este espacio, en la medida en que según los datos de 2019 (figura 8) existe una ligera supremacía de mujeres. No obstante, es necesario señalar que la discusión y el punto de partida no es la superioridad numérica de uno ni del otro, sino la igualdad de oportunidades para su consecuente paridad en este espacio y otros relacionados con la arqueología en el Perú.

Las cifras obtenidas - aunque expresan una menor participación de mujeres, y que creemos no reflejaría la cantidad de arqueólogas en el país y de aquellas que vienen a hacer arqueología en el Perú- son aspectos importantes de analizar en tanto se han creado estrategias que le permiten a la mujer desarrollarse profesional y académicamente. El gráfico de distribución por temas (figura 9) indica una importante participación de mujeres en contenidos vinculados a la arqueometría, ya que la estrategia desarrollada en este punto podría relacionarse con la idea de reforzar, a través de la incorporación de otras ciencias no sociales y humanas, los discursos y las explicaciones que directa o indirectamente pueden ser cuestionadas con mayor rigor e intencionalidad que las vertidas por los hombres, con lo cual se obtiene un trabajo «más científico» y «objetivo». De esta forma, las mujeres, aunque no todas, han encontrado un nicho que les permite su desarrollo académico.

A su vez, tanto mujeres como varones se mantienen en las tendencias estereotipadas. En el caso de los hombres, siguen siendo mayoría en temas que explican los grandes procesos, las propuestas teóricas y metodológicas, la dirección de proyectos, etc., todas consideradas, consciente o inconscientemente, a través del tiempo, «tópicos de hombres». Los temas de mujeres, por múltiples razones, se dirigen al análisis textil, actividad considerada exclusiva de mujeres, e incluso a la interpretación arqueológica, situación que ha cambiado como resultado de hallazgos arqueológicos que demuestran lo contrario, pero la praxis arqueológica 
mantiene este estereotipo. Es destacable que campos como la gestión del patrimonio, la arqueología histórica, arqueología de género y arqueología de contrato-rescate se conviertan en espacios compartidos por hombres y mujeres.

En general, la arqueología ha sido una ciencia iniciada por hombres y practicada ellos mismos durante mucho tiempo (Gero, 1988). Una consecuencia de lo anterior ha sido la interpretación sesgada del pasado y en la cual se invisibiliza el verdadero papel de la mujer en el pasado (Falcó, 2008; Conkey y Spector, 1984). Esta misma situación se volcó a la praxis de la disciplina. Pese a ello, notamos una creciente incursión de mujeres en la carrera. Así, nos encontramos ante la tendencia de que las mujeres entren a trabajar a este espacio tradicionalmente masculinizado. Para ello, la creación de estrategias, como los nichos académicos, suelen ser nuevas formas de establecer áreas donde la discriminación e invisibilización suelen ser menos percibidas.

Finalmente, consideramos que hoy el acto de exclusión explícita de las mujeres cada vez es menor en universidades y en espacios de investigación y divulgación. Por el contrario, se aprecia un proceso de crecimiento en la presencia de las mujeres en estas áreas. Creemos que existen mecanismos sutiles e implícitos que contribuyen a mantener y legitimar la segregación de las mujeres en general y las arqueólogas en particular. Al respecto, las cifras mostradas que reflejan las diferencias entre mujeres y hombres en las planas docentes (figura 3) y en el CNA (figura 6) obedecen a múltiples factores que deben ser buscados a través de otra clase de estudio, que permita recopilar esta información dentro de un marco general, es decir, de la praxis científica en el Perú, como también hallar las particularidades de la praxis arqueológica como parte del conjunto de ciencias. Esta debe estar guiada por preguntas como: «¿Cuáles son las condiciones de las arqueólogas en el campo laboral y académico?» 0 «¿Se encuentran en iguales condiciones?», entre otras que pueden ayudar a completar un análisis integral.

\section{Conclusiones}

Este artículo se encuadra dentro de la segunda perspectiva del estudio de género en arqueología, la praxis arqueológica, que se ocupa de las relaciones de género en la práctica arqueológica peruana. Por tanto, a partir de los datos e interpretaciones expuestas en secciones anteriores, concluimos que las relaciones de género dentro de la arqueología peruana experimentan un proceso de etapas drásticamente diferentes. La primera está caracterizada por una tendencia hacia la paridad y tiene que ver con el proceso de formación e incluso hasta el momento de finalización de ese 
proceso. Sin embargo, las otras etapas como la inserción laboral y la participación e investigación aún continúan reflejando actos de segregación, limitación y exclusión, aunque de manera menos explícita y drástica. Estas etapas, por tanto, muestran la existencia de un techo de cristal que no permite el progreso pleno de las mujeres en la disciplina, lo cual conlleva al desarrollo de estrategias que mitiguen esta situación. En este sentido, los llamados nichos académicos terminan por ser parte de este corpus estratégico en arqueología y en otras ciencias.

Finalmente, y como una reflexión, debemos indicar que se hace necesario y urgente que las universidades que ofrezcan la carrera de Arqueología, el Ministerio de Cultura, el Colegio de Arqueólogos y otros espacios relacionados con la arqueología, implementen políticas de género con miras a la instauración de espacios de paridad, de participación y competencia en igualdad de condiciones, y se creen medios e instituciones que fomenten y ayuden en la investigación tanto de mujeres como de hombres por igual.

\section{Contribuciones}

Contribución completa de la autora Lady Santana Quispe.

\section{Fuente de financiamiento}

Autofinanciado.

\section{Conflicto de intereses}

Ninguno, debido a que la autora no labora ni es parte de la entidad que publica el artículo. 


\section{REFERENCIAS BIBLIOGRÁFICAS}

Alcázar, L. y Balarin, M. (2018). Desigualdades en la academia: Mujeres en las ciencias sociales peruanas [Introducción]. En L. Alcázar y M. Balarin (Eds.), Desigualdades en la academia. Mujeres en las ciencias sociales peruanas (pp. 10-23). Lima: Grupo Sofía.

Agut, L. (2016). Género y ciencia avanzada: Análisis bibliométrico de la revista Nature desde una perspectiva de género (trabajo final de grado de Psicología, PS-1048). Universitat Jaume I, Castellón.

Bastida, C. (2015). Diferencias de género, el techo de cristal en España, dificultades de acceder a puestos de responsabilidad. (tesis de magíster). Universitat Jaume I, Castellón.

Bellelli, C., Berón, S. y Scheinsohn, V. (1993). Una arqueología de distinto género. Publicar en Antropología y Ciencias Sociales, 2(3), pp. 47-61.

Chacaltana, S. (2019). Mujeres e identidad de género en el Colesuyo. En L. Rosas (Ed.), Género y mujeres en la historia del Perú (pp. 27-56). Lima: Fondo Editorial de la Pontificia Universidad Católica del Perú.

Chacaltana, S. (2016). De los tambos incas a las tambarrías coloniales: economía colonial, legislación de tambos y actividades «licenciosas» de las mujeres indígenas. Boletín de Arqueología PUCP, 21, pp. 123-124.

Concytec (2017). I Censo Nacional de Investigación y Desarrollo a Centros de Investigación 2016. Recuperado de https://portal.concytec.gob.pe/index.php/publicaciones/censo-nacional-id

Conkey, M. (March 01, 2003). Has feminism changed archaeology? Journal of Women in Culture and Society, 28(3), pp. 867-880.

Conkey, M. y Spector, J. (1984). Archaeology and the study of gender. En M. Schiffer (Ed.), Advances in Archaeological Method and Theory, 7 (pp. 1-38). Cambridge: Academic Press.

Díaz-Andreu, M. (2013). Epílogo: últimas reflexiones y nuevas propuestas. En H. Tantaleán y C. Astuhuamán (Eds.), Historia de la arqueología en el Perú del siglo XX (pp. 603-616). Lima: IFEA-IAR.

Díaz-Andreu, M. (1998). Iberian post-Paleolithic art and gender: Discussing human representation in Levantine art». Journal of Iberian Archaeology, 0, pp. 33-51.

Falcó, R. (2003). La arqueología del género: Espacios de mujeres, mujeres con espacio. Recuperado de https://ieg.ua.es/es/documentos/publicaciones/ cuadernos-de-trabajos-de-investigacion/6-arqueologia-del-genero.pdf

Gastiazoro, M. (2013). Género y trabajo: mujeres en el Poder Judicial. Córdoba: Centro de Estudios Avanzados. 
Gero, J. (1988). Gender bias in Archaeology: Here, then and now. En S. Rosser (Ed.), Feminism within the Science and Health Care Professions: Overcoming Resistance (pp. 33-43). Oxford: Pergamon Press.

Guil, A. (2008). Mujeres y ciencia: Techos de cristal. EccoS Revista Científica, 10(1), pp. 213-23.

Heller, L. (2012). Diversidad de género en las organizaciones: Empresas globales, culturas locales (tesis doctoral). Universidad de Buenos Aires, Buenos Aires.

Ministerio de Cultura del Perú (2015). Actas del I Congreso Nacional de Arqueología. Recuperado de http://www.congresoarqueologia.cultura. gob.pe

Ministerio de Cultura del Perrú (2017). Actas del II Congreso Nacional de Arqueología. Recuperado de http://www.congresoarqueologia.cultura. gob.pe

Ministerio de Cultura del Perrú (2018). Actas del III Congreso Nacional de Arqueología. Recuperado de http://www.congresoarqueologia.cultura. gob.pe

Ministerio de Cultura del Perú (2015). Sumillas del IV Congreso Nacional de Arqueología. Recuperado de http://www.congresoarqueologia.cultura.gob.pe

Ministerio de Cultura del Perrú (2017). Sumillas del V Congreso Nacional de Arqueología. Recuperado de http://www.congresoarqueologia.cultura.gob.pe

Ministerio de Cultura del Perrú (2018). Sumillas del VI Congreso Nacional de Arqueología. Recuperado de http://www.congresoarqueologia.cultura.gob.pe

Novoa, P. (2013). Una aproximación a la obra de Rebeca Carrión Cachot entre 1947 y 1960. En H. Tantaleán y C. Astuhuamán (Eds.), Historia de la arqueología en el Perú del siglo XX (pp. 529-550). Lima: IFEA-IAR.

Nuñez, Claudia (23 de agosto de 2018). ¡H(ay) mujeres en la arqueología:dDinámica de género en la praxis arqueológica peruana! Simposio «Mujeres en la arqueología: pasado, presente y futuro contribuciones a la praxis arqueológica en el Perú». Museo Nacional de Antropología, Arqueología e Historia del Perú.

Peppino, A. (2016). Las mujeres y la ciencia en una sociedad patriarcal. I Congreso Iberoamericano de Ciencia, Tecnología, Sociedad e Innovación CTS+I.

Puertas, F. (2015). El papel de las mujeres en la ciencia y la tecnología. Madrid: Santillana. 
República del Perú (2014). Promulgación y publicación de la Ley Universitaria 30220. https://www.sunedu.gob.pe/wp-content/uploads/2017/04/ Ley-universitaria-30220.pdf

Sánchez Romero, M. (2000). Mujeres y espacios de trabajo en el yacimiento de los Castillejos (Montefrío). Arqueología Espacial, 22, pp. 93-106.

Sunedu (2019). Estadísticas de universidades por programa de estudio. Recuperado de https://www.sunedu.gob.pe/sibe/

Tavera, C. y Santana, L. (11 de octubre de 2018). Desigualdades impresas: un primer paso para el estudio de la mujer en la arqueología peruana. I Jornada de Estudios de Género y Mujeres en la Historia del Perú.

Recibido: 11/7/2019

Aceptado: 15/10/2019 